\title{
Systematic Analysis on Pipeline Leak Detection and Location
}

\author{
Yang Qibin \\ State Key Laboratory for Continental Dynamics \\ Department of Geology, Northwest University \\ Xi'an City, China \\ e-mail: yqb1008@126.com
}

\author{
Sun Wei \\ State Key Laboratory for Continental Dynamics \\ Department of Geology, Northwest University \\ Xi'an City, China \\ e-mail: sunwei5393901@vip.sohu.com
}

\begin{abstract}
Because pipeline has large pipe diameter, large throughout and high pressure, once pipeline leakage accident happens, the damage is quite serious. In addition, pipeline leakage accident caused by man-made drilling oil stolen every year results in huge economic losses on oilfield. Therefore, a real-time and accurate pipeline leak detection and location system not only can effectively decrease leakage loss and reduce the waste of manpower and material resources in patrolling work, but also is conductive to the management of oil pipeline and improvement of economic efficiency of enterprise. The paper determines leak detection and location project giving priority to negative pressure wave and supplemented by flow parameter analysis. The method not only can judge the accidence of leakage timely and accurately, but also can effectively avoid leakage false alarm caused by start or stop pumps in pipeline.
\end{abstract}

Keywords- pipeline leakage; detection;negative pressure wave

\section{CONVENTIONAL LEAKAGE DETECTION TECHNOLOGY OF NEGATIVE PRESSURE WAVE METHOD}

Negative pressure wave method is the method which is used widely on pipeline leakage detection and location internationally at present. It is based on signal processing. And the method doesn't need to establish hydraulic and mathematical model of pipeline, and most only use pressure signal. So the method adapts to the application of pipeline in China[1].

Because oil pipeline has the function of guiding wave, the negative pressure wave caused by leakage can transmit more than tens of kilometers along the pipeline. Its propagation velocity is equal to the propagation velocity of sound wave transferring fluid in pipeline. And the propagation velocity of negative pressure wave in the original oil pipeline is between 1000 meters and 1200 meters. As negative pressure wave has the characteristics of remote transmission, pressure transducer installed at the end and first of the pipeline can be used to capture negative pressure wave containing leakage information and to detect the leakage. After identifying leakage happens, leakage location can be made according to time difference of negative pressure wave caused by leakage transmitting to the head and end of the pipeline and negative pressure wave velocity[2].

Diagrammatic sketch that oil pipeline happens leakage is as follows:

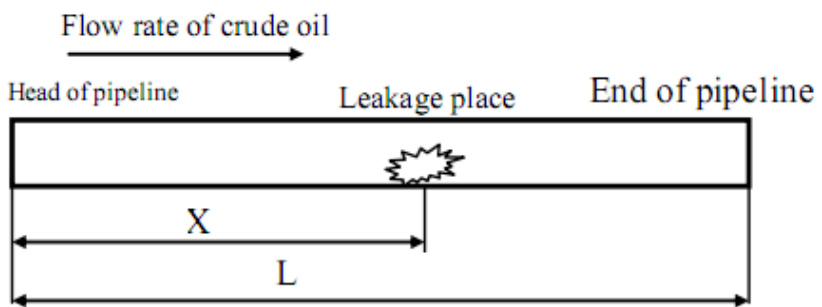

Figure 1 Diagrammatic sketch of pipeline leakage

Leak positioning formula of negative pressure wave method is:

$$
\frac{x}{v-v_{0}}-\frac{L-x}{v+v_{0}}=\Delta t=T_{1}-T_{2}
$$

And

$$
x=\frac{1}{2 v}\left[L\left(v-v_{0}\right)+\left(v^{2}-v_{0}^{2}\right) \Delta t\right]
$$

Because propagation velocity of negative pressure wave in crude oil is about between $1000 \mathrm{~m} / \mathrm{s}$ and $1200 \mathrm{~m} / \mathrm{s}$, and the normal flow velocity of fluid is about $1.5-3 \mathrm{~m} / \mathrm{s}, \mathrm{v}_{0}$ in the formula can be negligible. And the formula (2) can be simplified as:

$$
x=\frac{L+v \Delta t}{2}
$$

In the formula,

$\mathrm{x}$ : the distance of pipeline leakage position from the head of pipeline, meter;

$\mathrm{L}$ : the total length of pipeline, meter;

$\mathrm{T}_{1}$ : the time of negative pressure wave transmitting to the head of pipeline, second;

$\mathrm{T}_{2}$ : the time of negative pressure wave transmitting to the end of pipeline, second;

$\mathrm{v}$ : propagation velocity of negative pressure wave, $\mathrm{m} / \mathrm{s}$;

$\mathrm{v}_{0}$ : flow velocity of pipe-conveying medium, $\mathrm{m} / \mathrm{s}$;

$\Delta \mathrm{t}=\mathrm{T}_{1}-\mathrm{T}_{2}$

\section{LEAKAGE DETECTION AND LOCATION METHOD OF NEGATIVE PRESSURE WAVE FOR HOT OIL PIPELINE}

\section{A. Analysis of temperature along hot oil pipeline}

In the heating pipeline, the oil can continuously make its heat lost to the medium around pipeline with lower temperature after heating in the process of flowing along the pipeline, which can make the temperature of oil flow reduce 
constantly along the pipeline[3,4].Supposing the initial temperature of oil flow is $\mathrm{T}_{\mathrm{i}}{ }^{\circ} \mathrm{C}$, and the temperature of the medium around pipeline is $\mathrm{T}_{0}{ }^{\circ} \mathrm{C}$, the temperature of the heated oil flow has been reduced to $\mathrm{T}^{\circ} \mathrm{C}$ when the heated oil flow to the place from the start point. When oil flow begins to start from the place to go ahead for the pipeline with a short distance as $\mathrm{dl}$, the heat of oil flow dispersing is $\mathrm{dQ}=\mathrm{K}\left(\mathrm{T}-\mathrm{T}_{0}\right) * \pi^{*} \mathrm{D} * \mathrm{dl}$ in which $\mathrm{K}$ is total heat transfer coefficient of oil flowing surrounding medium. And if oil temperature reduced by $\mathrm{dT}^{\circ} \mathrm{C}$ after oil flows for the distance of $\mathrm{dl}$, the released heat of oil flow is $\mathrm{dQ}^{\prime}=-\mathrm{G}^{*} \mathrm{C}^{*} \mathrm{dT}$. Because the operation of $\mathrm{dl}$ and $\mathrm{dT}$ is opposite, negative sign exists.

According to heat balance theory, the released heat dQ of oil flow is equal to the heat dQ dispersing into surrounding medium. So thermal equilibrium relationship in the distance of $\mathrm{dl}$ is $[5,6]$ :

$$
\mathrm{K}\left(\mathrm{T}-\mathrm{T}_{0}\right) * \pi * \mathrm{D} * \mathrm{dl}=-\mathrm{G} * \mathrm{C} * \mathrm{dT}
$$

In the formula,

K:total heat transfer coefficient of oil flowing surrounding medium, $\mathrm{w} /(\mathrm{m} 2 \cdot \mathrm{k})$;

D:outside diameter of pipeline, $\mathrm{m}$;

$\mathrm{G}$ :mass flow of oil flow, $\mathrm{kg} / \mathrm{h}$;

$\mathrm{C}$ :specific heat capacity of crude oil, $\mathrm{kJ} / \mathrm{kg} \bullet \mathrm{k}$;

If the total heat transfer coefficient $\mathrm{K}$ is constant, and the oil temperature reduces to $\mathrm{T}_{\mathrm{x}}{ }^{\circ} \mathrm{C}$ after flowing the pipeline with the distance as $\mathrm{X}$, the integration along the pipeline from $\mathrm{o}$ to $\mathrm{x}$ is:

$$
\int_{0}^{X} K \pi d l=\int_{T_{i}}^{T_{X}}-G C \frac{d T}{T-T_{0}}
$$

And the solution is:

$$
T_{X}=T_{0}+\left(T_{i}-T_{0}\right) e^{\frac{-K \pi D X}{G C}}
$$

Formula (6) indicates that the law of temperature drop of oil flow in hot oil pipeline is a curve changing on the basis of index law, and that temperature gradient everywhere is different. The temperature at the entrance of the heated pipeline is higher, and oil flow and surrounding medium have large temperature difference, rapid heat dissipation and fast temperature drop. But as the oil temperature at the exit of the heated pipeline has low oil temperature and little temperature difference, temperature drop is slow.

In addition, because friction resistance makes pressure reduce constantly in the process of oil flowing along the pipeline, the friction loss converts into friction heat, which heats oil flow. But the change of oil temperature made by frictional resistanceis little compares with the change of oil temperature brought by heat dissipation along pipeline, so it can be negligible. The parameters of $\mathrm{D}, \mathrm{G}, \mathrm{C}, \mathrm{T}_{\mathrm{i}}$ and $\mathrm{T}_{0}$ of oil temperature calculating oil temperature in formula (6)is based on the practical and applied pipeline to determine concrete values.

\section{B. Determination on wave velocity of negative pressure in hot oil pipeline}

Wave velocity of negative pressure in oil pipeline is determined by elasticity and density of piped oil and elasticity of tube and pipe. Coefficient of cubical elasticity for crude oil is different with the difference of variety, temperature and pressure. Wave velocity formula of negative pressure in isothermal transportation is $[6,7,8]$ :

$$
v=\sqrt{\frac{K / \rho}{1+\frac{K D C_{1}}{E e}}}
$$

In the formula,

$\mathrm{v}$ : propagation velocity of negative pressure in pipeline, $\mathrm{m} / \mathrm{s}$;

$\mathrm{K}$ :coefficient of cubical elasticity for crude oil, $\mathrm{Pa}$;

$\rho:$ density of crude oil, $\mathrm{kg} / \mathrm{m}^{3}$;

E:elasticity modulus of tube and pipe, $\mathrm{Pa}$;

D:inside diameter of pipeline, $\mathrm{m}$;

e:wall thickness of pipeline, $\mathrm{m}$;

$\mathrm{C}_{1}$ :correction factor relating to pipe constraint conditions, it is dimensionless.

But in heat transportation, because the parameters of $\mathrm{K}$ and $\rho$ in formula (7) are influenced greatly by temperature, wave velocity formula of negative pressure in hot oil pipeline should be changed into [7]:

$$
v(t)=\sqrt{\frac{K(t) \rho(t)}{1+\frac{K(t) D C_{1}}{E e}}}
$$

According to the characteristics of crude oil in our country, the following are the relation between the parameters and temperature influenced by temperature in formula (8) and the value of constant in formula.

(1) Density of crude oil, $\rho$ : density of crude oil is almost not influenced by density of crude oil and is greatly influenced by temperature. Transformation formula of density with temperature is [5]:

$$
\rho(t)=\rho_{20}-\xi(t-20)
$$

In the formula,

$\rho(\mathrm{t})$ : density of crude oil in $\mathrm{t}^{\circ} \mathrm{C}, \mathrm{kg} / \mathrm{m}^{3}$;

$\rho_{20}$ : density of crude oil in $20^{\circ} \mathrm{C}, \mathrm{kg} / \mathrm{m}^{3}$;

$\xi$ :coefficient of cubic expansion for crude oil, $\xi=1.825$ $0.001315 \rho 20, \mathrm{~kg} /\left(\mathrm{m}^{3} \cdot{ }^{\circ} \mathrm{C}\right)$;

(2) Coefficient of cubical elasticity for crude oil, K: it represents the feature of fluid volume changing caused by the change of ambient pressure. Liquid coefficient of cubical elasticity of hydrocarbon which is the main component part of crude oil is influenced greatly by the temperature, as shown in Table 1 which indicates that the higher the temperature, the smaller the coefficient of cubical elasticity. The value of fluid coefficient of cubical elasticity $\mathrm{K}$ is equal to reciprocal value of compression coefficient. Calculation formula of compression coefficient is as follows [7]:

$$
\ln \left(F \times 10^{10}\right)=0.51992+0.0023662 t+846596 / \rho_{0}^{2}
$$

In the formula:

$\mathrm{F}$ : compression coefficient,Pa- 1 ;

$\rho_{0}$ : standard density, $\rho_{0}=\rho_{4}{ }^{20}, \mathrm{~kg} / \mathrm{m}^{3}$;

$\mathrm{t}$ :temperature, ${ }^{\circ} \mathrm{C}$;

We can get elasticity coefficient $K$ from $K=1 / F$. 
TABLE I. COEFFICIENT OF CUBICAL ELASTICITY OF MAIN COMPONENT IN CRUDE OIL [9]

\begin{tabular}{|c|c|c|c|c|c|}
\hline \multirow{2}{*}{$\begin{array}{c}\text { Liquid } \\
\text { name }\end{array}$} & \multicolumn{5}{|c|}{ Coefficient of cubical elasticity $\left(\times 10^{5} \mathrm{~Pa}\right)$} \\
\hline & $20^{\circ} \mathrm{C}$ & $30^{\circ} \mathrm{C}$ & $40^{\circ} \mathrm{C}$ & $50^{\circ} \mathrm{C}$ & $60{ }^{\circ} \mathrm{C}$ \\
\hline propane & 1760 & 1370 & 1040 & 715 & \\
\hline butane & 3560 & 3020 & 2510 & 2130 & \\
\hline gasoline & 9060 & & & 7600 & \\
\hline kerosene & 13600 & & 12050 & & \\
\hline lube oil & 15600 & & & 13000 & \\
\hline water & 23900 & & 22150 & & 21750 \\
\hline
\end{tabular}

(3) Correction coefficient, $\mathrm{C}_{1}$ : literature [9] points out that the constraint conditions can be divided into three categories according to 3 different support situations of pipeline:

1) the upstream of pipeline is fixed and the downstream is flexible freely.

2)both ends of pipeline are fixed, which limits displacement of axial direction.

3 ) the pipeline is connected by many expansion points.

Relative to the three constraint conditions, the values of $\mathrm{C}_{1}$ is is respectively:
1) $C_{1}=1-\frac{\mu}{2}$
2) $C_{1}=1-\mu^{2}$
3) $C_{1}=1$

$\mu$ is poisson's coefficient of tube and pipe. As the oil pipeline studied in the paper is buried under the ground deeply, the buried steel tube is restricted by soil resistance reduction and anchor block, which belongs to the second situation.

(4) elasticity modulus of tube and pipe E, inner diameter of pipe D and wall thickness e: there are several elasticity modulus and poisson's coefficients of conventional materials in Table 2 .

TABLE II. ELASTICITY MODULUS AND POISSON'S COEFFICIENTS OF CONVENTIONAL MATERIALS

\begin{tabular}{c|c|c}
\hline Name & $\boldsymbol{E}\left(\times \mathbf{1 0}^{\mathbf{9}} \mathbf{P a}\right)$ & $\boldsymbol{\mu}($ dimensionless $)$ \\
\hline nodular cast iron & 165.5 & 0.28 \\
brass & 103.4 & 0.34 \\
copper & 110.3 & 0.28 \\
steel & 206.9 & 0.30 \\
aluminum & 72.4 & 0.33 \\
polythylene & 2.76 & 0.45 \\
concrete & $30-108$ & $0.08-0.18$ \\
asbestos cement & 23.4 & 0.30 \\
rubber & 0.07 & 0.45 \\
\hline
\end{tabular}

The studied oil pipeline in the paper is manganese steel according to Table 2 . Elasticity modulus E is $206.9 \times 10^{9} \mathrm{~Pa}$, poisson's coefficient $\mu$ is 0.3 and pipeline specification is $\Phi 273 \times 7 \mathrm{~mm}$.

\section{Determination on location method of negative pressure in hot oil pipeline}

The conclusion that the temperature of crude oil changes with conveying distance is drawn in chapter B. And the change law is shown in Formula (6).

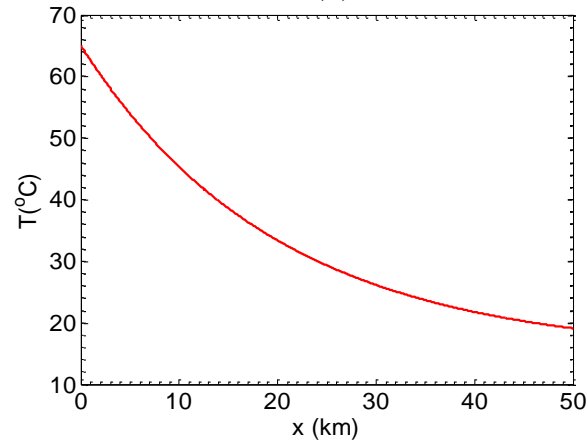

Figure 2. Curve of crude oil temperature changing with distance

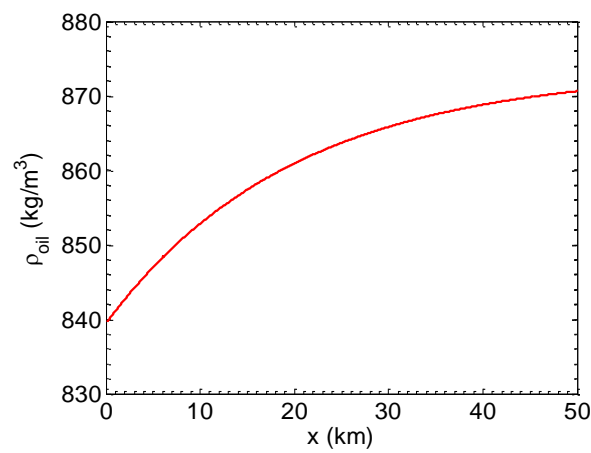

Figure 3. Curve of crude oil density changing with distance

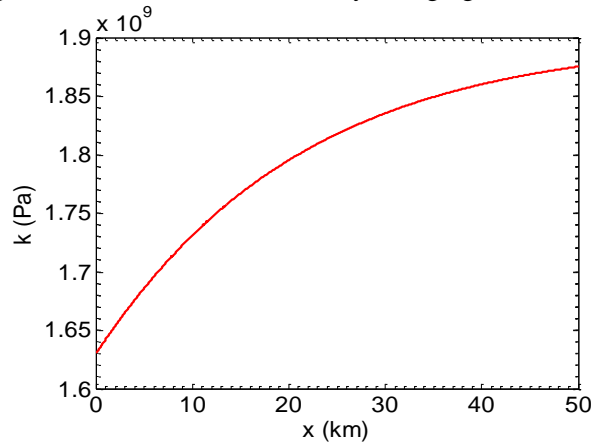

Figure 4. Curve of coefficient of cubical elasticity for crude oil changing with distance

Formula (6) is respectively substituted into formula (9) and (10), which can get the expression that the parameter $\mathrm{K}$ and $\rho$ in the distance $\mathrm{X}$ of crude oil transportation, that is $\mathrm{K}(\mathrm{x})$ and $\rho(x)$. And the curve of temperature $T$, density $\rho$ and coefficient of cubical elasticity $\mathrm{K}$ changing with transporting distance $\mathrm{X}$ is respectively shown in Figure 2, 3 and 4.

The expression of $\mathrm{K}(\mathrm{x})$ and $\rho(\mathrm{x})$ is respectively substituted into formula (8), and we can get relational expression between negative pressure wave velocity and crude oil transporting distance, $\mathrm{v}(\mathrm{x})$.

$$
v(x)=\sqrt{\frac{K(x) / \rho(x)}{1+\left(K(x) D C_{1} / E e\right)}}
$$

Its variation trend along the pipeline is shown in Figure 5. 
If negative pressure wave velocity is changed into the form $\mathrm{v}(\mathrm{x})$ of changing with distance, the needed time of negative pressure wave generated in leakage point $\mathrm{X}_{\mathrm{L}}$ transporting to the head and end of pipeline is respectively:

$$
t_{1}=\int_{0}^{x_{L}} \frac{1}{v(x)} d x, t_{2}=\int_{x_{L}}^{L} \frac{1}{v(x)} d x
$$

And the leak positioning formula of negative pressure wave can be written as:

$$
\Delta t=t_{1}-t_{2}=\Delta t\left(x_{L}\right)=\int_{0}^{x_{L}} \frac{1}{v(x)} d x-\int_{x_{L}}^{L} \frac{1}{v(x)} d x
$$

Figure 5. Curve of negative pressure wave velocity varying with distance

$\Delta t$ is time difference of negative pressure wave transporting to the head and end of the pipeline and is determined by the difference of $t_{1}$ and $t_{2}$ for negative pressure wave transporting to the head and end of the pipeline. $\mathrm{x}_{\mathrm{L}}$ is the leakage point position needing to be solved.

As shown in formula (13), $\Delta t\left(x_{L}\right)$ is the function changing with the leakage point position $\mathrm{x}_{\mathrm{L}}$. Further analysis indicates that $\Delta t\left(x_{L}\right)$ is a monotonous function. The function is considered.

$$
y\left(x_{L}\right)=\left|\Delta t\left(x_{L}\right)-\Delta t_{0}\right|
$$

In the formula, $\Delta t_{0}$ is the time difference of negative pressure wave transporting to the head and end of the pipeline obtained by measurement. As arc..., is monotonous, only when $\mathrm{x}_{\mathrm{L}}=\mathrm{x}_{\mathrm{L} 0}, y\left(x_{L}\right)$ can get the minimum value 0 . Therefore, the problem of solving the leakage point position $\mathrm{x}_{\mathrm{L}}$ can be converted into the problem of solving function (14).

\section{ApPlication of Trapezoidal RULE ON NEGATIVE PRESSURE WAVE Position IN HOT OIL PIPELINE.}

Engineering technology often needs integral calculation. In theory, as for the calculation of definite integral of continuous function $f(x)$ in [a,b], it only needs to find out the original function $\mathrm{F}(\mathrm{x})$ of $f(x)$, and it is easy to solve the definite integral value according to Newton-Leibniz formula (15).

$$
\int_{a}^{b} f(x)=F(b)-F(a)
$$

But in practical application, because the presentation format of the integrand $f(x)$ in very complicated, for example, the negative pressure wave velocity $\mathrm{v}(\mathrm{x})$ in the paper is a very complicated function, and its original function can't use be expressed by limited form of elementary function, the definite integral of formula (13) can't be solved by analytical method like formula (15) which used Newton-Leibniz formula. Therefore, it is necessary to calculate with an approximate method, that is, numerical integration method.

The significance of formula (15) solving definite integral geometrically is the area of trapezoid with curve $y=f(x)$, line $x=a, x=b$ and $x$ axis. Therefore, no matter the integrand is given with any form and if the original function can be found out, only calculating approximately the area of corresponding trapezoid with curve side can solve the approximate value of the definite integral, which is the basic thought of numerical integration.

Numerical integration method includes trapezoidal method, Gauss's processes and Simpson's formula. We can see from Figure 5 that the variation trend of the integrand is relatively slow. So the paper adopts numerical integration of trapezoidal method to realize the solution of integration.

\section{A. Numerical integration of trapezoidal method}

Considering the definite integral of any univalent function $f(x)$ in $(a, b)$, the $(a, b)$ is divided into $n$ evenly spaced lattice with width as $\mathrm{h}$, and the corresponding coordinate is $\left(\mathrm{x}_{1}, \mathrm{x}_{2}, \mathrm{x}_{3}, \ldots, \mathrm{x}_{\mathrm{n}}\right)$.

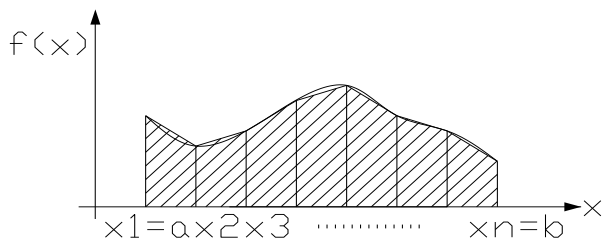

Figure 6. Numerical integration of trapezoidal method

So integral value of $f(x)$ in any lattice $\left(x_{i}, x_{i+1}\right)$ can be expressed as :

$$
\int_{x i}^{x_{i+1}} f(x) \mathrm{d} x=\frac{h}{2}\left[f\left(x_{i}\right)+f\left(x_{i+1}\right)\right]+R(f)
$$

And the integration of $f(x)$ in the interval $(a, b)$ can be expressed as :

$$
\begin{aligned}
\int_{a}^{b} f(x) \mathrm{d} x & =\int_{x_{0}}^{x_{1}} f(x) d x+\int_{x_{1}}^{x_{2}} f(x) d x+\cdots+\int_{x_{n-1}}^{x_{n}} f(x) d x \\
& =\sum_{i=0}^{n-1} \int_{x_{i}}^{x_{i+1}} f(x) d x \\
& \approx \sum_{i=0}^{n-1} \frac{x_{i+1}-x_{i}}{2}\left[f\left(x_{i}\right)+f\left(x_{i+1}\right)\right] \\
& =\frac{h}{2} \sum_{i=0}^{n-1}\left[f\left(x_{i}\right)+f\left(x_{i+1}\right)\right] \\
& =\frac{h}{2}\left[f\left(x_{0}\right)+2 \sum_{i=1}^{n-1} f\left(x_{i}\right)+f\left(x_{N}\right)\right]+R_{n}(f) \\
& =\frac{h}{2}\left[f\left(x_{0}\right)+f\left(x_{N}\right)\right]+h\left[f\left(x_{2}\right)+\cdots+f\left(x_{N-1}\right)\right]+R_{n}(f)
\end{aligned}
$$


In the formula, $R_{n}(f)$ is truncation error for numerical integration of trapezoidal method, and the error order is $R_{n}(f)=O\left(h^{2}\right)$.

$$
R_{n}(f)=-\frac{b-a}{12} h^{2} f^{\prime \prime}(\eta), \eta \in(a, b)
$$

In the condition that the function has no singularity, the numerical integral value obtained in formula (17) can achieve very high accuracy. Meanwhile, it should be pointed out that the accuracy of integrant depends on the size of the number of the divided lattice $\mathrm{n}$. When $\mathrm{n}$ increases, integral value can approximate to actual value. But in general, when $\mathrm{n}$ increased to a certain numerical value, integral value will be stable gradually.

According to formula (17), $\Delta t\left(x_{L}\right)$ in formula (13) and (14) can be solved according to the numerical integration of trapezoidal method.

$$
\begin{aligned}
\Delta t\left(x_{L}\right) & =\int_{0}^{x_{L}} \frac{1}{v(x)} d x-\int_{x_{L}}^{L} \frac{1}{v(x)} d x \\
& =\frac{h}{2}\left[f\left(x_{0}\right)+f\left(x_{2}\right)+\cdots+f\left(x_{N-1}\right)+f\left(x_{N}\right)\right] \\
& =\frac{h}{2} \sum_{i=0}^{x_{L}-1}\left[\frac{1}{v\left(x_{i}\right)}+\frac{1}{v\left(x_{i+1}\right)}\right]-\frac{h}{2} \sum_{i=x_{L}}^{L}\left[\frac{1}{v\left(x_{i}\right)}+\frac{1}{v\left(x_{i+1}\right)}\right]
\end{aligned}
$$

\section{B. Realization of numerical integration of trapezoidal method on positioning of leakage point}

We can know from the above-mentioned theoretical analysis that the process of realizing positioning of leakage point is very complicated. In order to guarantee the implementation of the algorithm [5-6], MATLAB software is used to write the specific procedures in the paper. MATLAB is a powerful technology application software developed by Mathworks in America and has high-efficiency mathematical calculation ability and drawing skill. Under the situation of MATLAB, the task uses the corresponding function in mathematical calculation tools, and the solution of leakage point position is realized by program. Numerical integration of trapezoidal method described in formula (19) can be realized by using the function trapz( ) in MATLAB software. The function trapz ( ) has the analytical expression without the need to know integral function. The corresponding equinoctial function value can be obtained by interpolation and fitting curve method, and the characteristics of integrant solution can be realized by composite trapezoidal integral method[7,8].

In addition, there are many methods for the determination on leakage point position $x_{L}$ in formula (19) such as search method based on genetic algorithm and point-to-point comparison. The task in the paper also applies the method of searching for the optimal solution to get leakage point $x_{L}$. And the concrete realization is to use MATLAB to optimize the function fminbnd( ) in toolkit[9].

The ideal of the method of searching for the optimal solution is to make $x_{L}$ change with some trend and to observe which value $x_{L}$ takes can make formula (14) get the minimum value, that is, the time difference calculated by formula (19) is near to the time difference of negative pressure wave reaching the head and end of pipeline obtained by actual calculation, and $x_{L}$ is the searched optimal solution.

According to the thought of searching for the optimal solution, the concrete process of applying the search algorithm of the function fminbnd( ) is that golden section method and quadratic interpolation are used in the given interval, and the optimal local minimum solution is given after iterative operation with limited times. It needs to notice that fminbnd( ) is the function solving the minimum of function of one variable in the given interval, that is, it is allowed to have only one input variable which is distance $\mathrm{x}$. But we can see from the former analysis that the solution of the minimum for $\left|\Delta t\left(x_{L}\right)-\Delta t_{0}\right|$ in formula (14) not only needs distance $\mathrm{x}$, but also needs negative pressure wave velocity $\mathrm{v}(\mathrm{x})$. In order to make $\mathrm{v}(\mathrm{x})$ input the function fminbnd( ), nested function is used to nest it, which can realize that the minimum of $\left|\Delta t\left(x_{L}\right)-\Delta t_{0}\right|$ can be found out in the given interval (nested function is the special function quality of MATLAB7 edition).

As there are many parameters and calculations in the program, the calculation on $\mathrm{K}(\mathrm{x}), \rho(\mathrm{x})$ and numerical integration, the search of the minimum is respectively written into subroutine for invocation to keep that the program is neat and east to read. The setup of each setting parameter is in the beginning part of the main program. When the environment of the applied pipeline varies, it is easy to be modified.

\section{Precision analysis on numerical integration of trapezoidal method}

According to the former analysis, the leakage point position is $\mathrm{x}_{\mathrm{L}}$ making the function $\mathrm{y}\left(\mathrm{x}_{\mathrm{L}}\right)$ in formula (14) get the minimum value, so using the numerical integration method to solve $\Delta t\left(x_{L}\right)$ is the key to the precision of leak location. Therefore, it is necessary to analyze the precision of numerical integration.

(1) Precision analysis on numerical integration

Studying the precision of integration needs to compare with precise theoretical value. As the expression of negative pressure wave $\mathrm{v}(\mathrm{x})$ (formula (11)) is complicated, it is difficult to get the precise expression of $\Delta t\left(x_{L}\right)$ (formula (13)) . In order to solve the problem, the method of fitting of a polynomial is used to get the expression which approximates to be analysable for wave velocity of negative pressure wave, and then obtain the analytical expression of time difference $\Delta t\left(x_{L}\right)$ which is as the reference for verifying the precision of numerical integration of trapezoidal method and location. Under the premise with accuracy, second-order polynomial is applied for fit to make the problem keep simplified, and the analytical expression of supposing the wave velocity of negative pressure wave is:

$$
v(x)=a_{1} x^{2}+a_{2} x+a_{3}
$$

In the expression, the coefficients $a_{1}, a_{2}$, and $a_{3}$ are determined by least squares in fitting process (the concrete 
fitting process is realized by the program in MATLAB). And the analytical expression of integration in formula (13) is obtained.

$$
\int \frac{1}{v(x)} \mathrm{d} x=\frac{2}{\sqrt{4 a_{1} a_{3}-a_{2}^{2}}} \arctan \left(\frac{2 a_{1} x+a_{2}}{\sqrt{4 a_{1} a_{3}-a_{2}^{2}}}\right)
$$

The fitting effect is shown in the following figure:

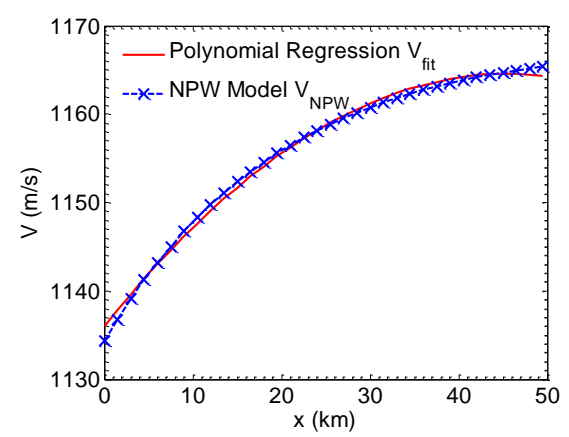

Figure 7. Binomial fitting effect on negative pressure wave velocity

Solid line in the figure is the actual negative pressure wave velocity. The dotted line and $\mathrm{X}$ sign are binomial fitting result on negative pressure wave velocity.

We can see from figure (7) that the fitting curve described in formula (20) has high anastomosis with actual negative pressure wave curve. The following is a qualitative analysis on the expression of making the fitting function as negative pressure wave. The values of a series of leakage point $\mathrm{x}_{\mathrm{L}}$ are set in the interval $(0, \mathrm{~L})$, numerical integration of trapezoidal method is used to respectively calculate the time difference $\Delta t\left(x_{L}\right)$ which is then compared with the time difference $\Delta t\left(x_{L}\right)$ obtained by calculation in formula (21), and we can know the precise condition of numerical integration of trapezoidal method. The concrete method is that in the process of numerical integration, the interval $(0, \mathrm{~L})$ is divided into $\mathrm{N}=1000$ parts, and trapezoidal method is used for numerical integration. As shown in Figure 8, the time difference obtained by numerical integration of trapezoidal method accords with solution value. Red curve in the figure is the result of numerical integration of trapezoidal method, blue curve and $\mathrm{x}$ represent the result achieved according to the analysis.

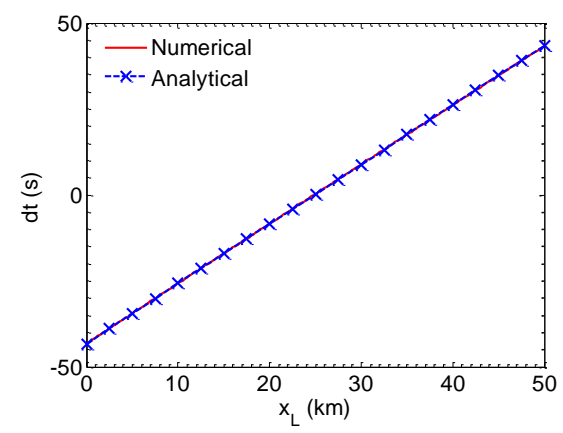

Figure 8. Variation condition of time difference $\Delta \mathrm{t}(\mathrm{xL})$ with leakage point $X_{\mathrm{L}}$

Figure 9 is the curve relative precision changing with leakage point position. And the relative precision is calculated by the following formula:

$$
\text { Relative Error }=\left|\frac{d t-d t_{a}}{d t}\right|
$$

In the formula, dt is the time difference obtained by numerical integration of trapezoidal method in formula (13), and dta is the resolution value calculated out in formula (21) We can see that the integration precision is less than $5 \times 10^{-9}$ in most leakage points, and fluctuation appears only in the place next to the center ( $\mathrm{dt}$ is very small 0 and the integration precision is less than $10^{-7}$.

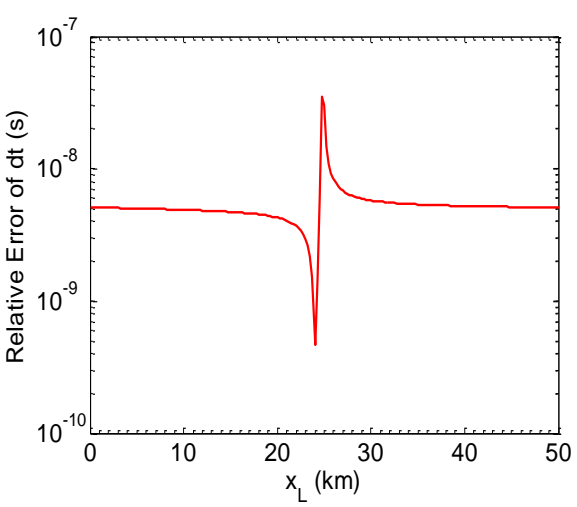

Figure 9. Relative error of numerical integration of trapezoidal method

(2) Precision analysis on location

From the former theoretical analysis, we can find that applying numerical integration of trapezoidal method can realize the calculation of high accuracy $\Delta t\left(x_{L}\right)$. As for a leakage point location system, we are more concerned with the precision of leakage point location which is analyzed in the following part. The task uses Golden Search method t to realize the determination on leakage point position by program. In order to study the precision of leakage point location, we firstly suppose that the time difference of the negative pressure wave obtained by measurement transporting to the head and end of the pipeline is 10 seconds, and it is taken into the leakage point position to find out leakage point $\mathrm{X}_{\mathrm{L}}$ by searching for subroutine, and then the value of $X_{L}$ is substituted into formula (21) for getting the corresponding time difference $\Delta t$. We can clearly see the location precision of leakage point searching for subroutine by analyzing relative error.

$$
\text { Location Relative Error }=\left|\frac{\Delta t-\Delta t_{0}}{\Delta t}\right|
$$

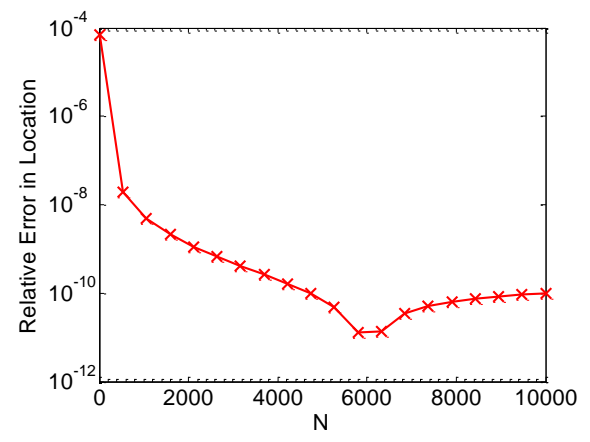

Figure 10 .Condition of location precise changing with the division number $\mathrm{N}$ of integral interval 
Figure 10 is the rule of located relative error changing with the division number $\mathrm{N}$ of integral interval. We can find that the precision of leakage location is higher, and the location error tends to saturation slowly with the increase of $\mathrm{N}$. Therefore, the value of $\mathrm{N}$ should be selected appropriately in actual situation, which not only can ensure the accuracy, but also reduces time redundancy brought by unnecessary calculation.

\section{CONCLUSION}

Combined with the characteristic of oil pipeline needing heating transportation in our country, the paper makes improvement on conventional formulas of leakage location for negative pressure wave. But the improved expressions of negative pressure wave are very complicated, and it is difficult to get analytical solution in leakage location formula, so the task introduces numerical integration of trapezoidal method after analyzing the characteristics of negative pressure wave function, and uses MATLAB to realize concrete numerical calculation and optima process of leakage position. And the paper makes qualitative analysis and verification on the precision and accuracy of the numerical integration method.

\section{REFERENCES}

[1] Haitao Xiong, Yufeng Ren, Pan Jia, "A Novel Classification Approach for C2C E-Commerce Fraud Detection", JDCTA: International Journal of Digital Content Technology and its Applications, Vol. 7, No. 1, pp. 504 -511, 2013

[2] Meipeng Ren, Xiangfang Li, Xiaoliang Chen, Weina Ren, "Research of Drilling Gas Kick and Blowout Characteristic Based on The Volume of Fluid Model", JDCTA: International Journal of Digital Content Technology and its Applications, Vol. 7, No. 1, pp. 797 -804, 2013

[3] JIAO Hongqiang, JIA Limin, JIN Yanhua, "A New Network Intrusion Detection Algorithm based on Radial Basis Function Neural Networks Classifier", AISS: Advances in Information Sciences and Service Sciences, Vol. 4, No. 1, pp. 170 -176, 2012

[4] Jing Shen, Muqing Wu, Linlin Luan, "An Improved Semi-blind Joint Data Detection and Channel Estimation Algorithm for MIMO-OFDM System", AISS: Advances in Information Sciences and Service Sciences, Vol. 4, No. 1, pp. 371- 380, 2012

[5] Xiaohong Long, Jing Zhou, "An Efficient Method For Dark License Plate Detection", JDCTA: International Journal of Digital Content Technology and its Applications, Vol. 7, No. 1, pp. 477- 485, 2013

[6] Wei Zhao, Zhoumo Zeng, Shixu Guo, Shili Chen, Yu Zhang, "Pipeline leakage monitoring system based on multi-layer network communication", JDCTA: International Journal of Digital Content Technology and its Applications, Vol. 6, No. 7, pp. 197-205, 2012

[7] Hui Li, "Multi-Scale Hermitian Wavelet Order Envelope Spectrum Based Bearing Fault Detection and Diagnosis", JDCTA: International Journal of Digital Content Technology and its Applications, Vol. 7, No. 1, pp. $440-449,2013$

[8] ZHANG Wei, LIU Xiaoyuan, QI Ruolong, Jiang Yong, "Improved Locally Linear Embedding Based Method for Nonlinear System Fault Detection", IJACT: International Journal of Advancements in Computing Technology, Vol. 5, No. 1, pp. 515- 523, 2013

[9] Dunqiu Fan, "The Development Mode of Marginal Oilfield Based on Jack-Up Production Platform with Oil Cabins", JCIT: Journal of Convergence Information Technology, Vol. 8, No. 1, pp. 382 - 387, 2013 